\title{
Schottky-barrier lowering in silicon nanowire field-effect transistors prepared by metal-assisted chemical etching
}

\author{
M. Zaremba-Tymieniecki and Z. A. K. Durrani ${ }^{\text {a) }}$ \\ Department of Electrical and Electronic Engineering, Imperial College London, South Kensington Campus, \\ London SW7 2AZ, United Kingdom
}

(Received 4 January 2011; accepted 23 February 2011; published online 10 March 2011)

\begin{abstract}
We investigate the influence of Schottky barrier lowering in Si nanowire field-effect transistors, using nanowires prepared by metal-assisted chemical etching. The experimental electrical characteristics of a $p$-channel transistor are modeled using thermionic emission of holes across the reverse-biased source Schottky barrier. This barrier is lowered by the image-force potential, and by the electric field generated by both source-drain and gate voltages. The gate voltage lowers the barrier height directly and in addition, modulates the effect of the source-drain voltage on barrier lowering. (C) 2011 American Institute of Physics. [doi:10.1063/1.3565971]
\end{abstract}

Silicon nanowires (SiNWs) have, in recent years, shown great promise for the development of nanoelectronic, thermoelectric, solar cell, and biological/chemical sensing devices. ${ }^{1-5}$ In particular, SiNWs have attracted interest for high-performance field-effect transistor (FET) applications. ${ }^{1,2}$ Here, a SiNW forms the FET channel and provides a means to obtain good gate-control with high scalability. SiNW FETs are also, in principle, compatible with large-scale integrated processes. However, in many cases, Schottky barriers (SBs) exist at the metal contacts to the $\mathrm{SiNW},{ }^{2}$ forming a SB FET. The gate voltage then modulates the SB and strongly influences the FET characteristics.

SiNWs with minimum diameters $<10 \mathrm{~nm}$, and lengths up to $\sim 100 \mu \mathrm{m}$, are often prepared by vapor-liquid solid growth or high-resolution lithographic techniques. ${ }^{6,7}$ More recently, SiNWs have been prepared by metal assisted chemical etching ${ }^{4,8}$ (MACE) of Si wafers. Here, electroless deposition of a silver dendritic network on a Si wafer catalyzes subsequent chemical etching of a vertical, densely packed SiNW array. SiNWs with diameter from $\sim 20$ to 300 $\mathrm{nm}$ and length up to $\sim 150 \mu \mathrm{m}$ have now been demonstrated for FET, thermoelectric, and solar cell applications. ${ }^{4,9,10}$ In comparison with other preparation techniques, the MACE process is low cost and only requires wet-etching of a $\mathrm{Si}$ wafer. Furthermore, SiNWs may be formed over the entire wafer surface, ${ }^{9}$ with density $\sim 10^{9} / \mathrm{cm}^{2}$.

In this paper, we demonstrate that the lowering of a reverse-biased SB may be used to explain the electrical characteristics of FETs using SiNWs prepared by MACE. The $\mathrm{SBs}$ are formed using $\mathrm{Ti}_{\mathrm{x}} \mathrm{Si}_{\mathrm{y}} / \mathrm{Al}$ silicide contacts. We find that in $p$-channel FETs, the experimental $I_{S D^{-}} V_{S D}$ sourcedrain output characteristics, at various gate voltage $V_{G}$, may be modeled using thermionic emission of holes across the reverse-biased source SB. This barrier is lowered by the image-force potential, and by the local electric field caused by $V_{S D}$ and $V_{G}$. We find that once $I_{S D}$ begins to saturate, SB lowering is sufficient to explain both the drain and gate voltage dependence of the characteristics.

Our SiNWs were synthesized as follows. A [100] Si sample ( $p$-type, doped with boron at $10^{18} / \mathrm{cm}^{3}$ ) was immersed in hydrofluoric acid (HF) $/ \mathrm{AgNO}_{3}$ solution at room

${ }^{a)}$ Electronic mail: z.durrani@imperial.ac.uk. temperature for $5 \mathrm{~min}$. In solution, several processes occur simultaneously. Ag particles deposit on the substrate by electroless metal deposition, a network of $\mathrm{Ag}$ dendrites grows from these particles, and the Si substrate is etched. Etching is accelerated using nitrate ions as an oxidizing agent. Left for $2 \mathrm{~h}$, this produces a densely packed, vertical, SiNW array with NWs $\sim 150 \mu \mathrm{m}$ long. $\mathrm{HNO}_{3}$ is then used to remove the Ag. Figure 1(a) shows a scanning electron micrograph of the SiNW array, where SiNW diameters vary from 40 to $300 \mathrm{~nm}$ and the NW density is $\sim 10^{9} / \mathrm{cm}^{2}$. The SiNWs can be extremely long, with maximum aspect ratio $\sim 3000$. Figure 1(b) shows a transmission electron micrograph of a SiNW. The NW surface roughness is $\sim 1-5 \mathrm{~nm}$, and there is a $\sim 5$-nm-thick surface oxide layer. After etching, the SiNWs can be dispersed in isopropyl alcohol (IPA) using ultrasonic agitation.

Our SiNW $p$-FETs, shown schematically in Fig. 1(c), are fabricated as follows. A $p$-type $\mathrm{Si}$ wafer with a thermallygrown 150-nm-thick oxide layer forms the device substrate,
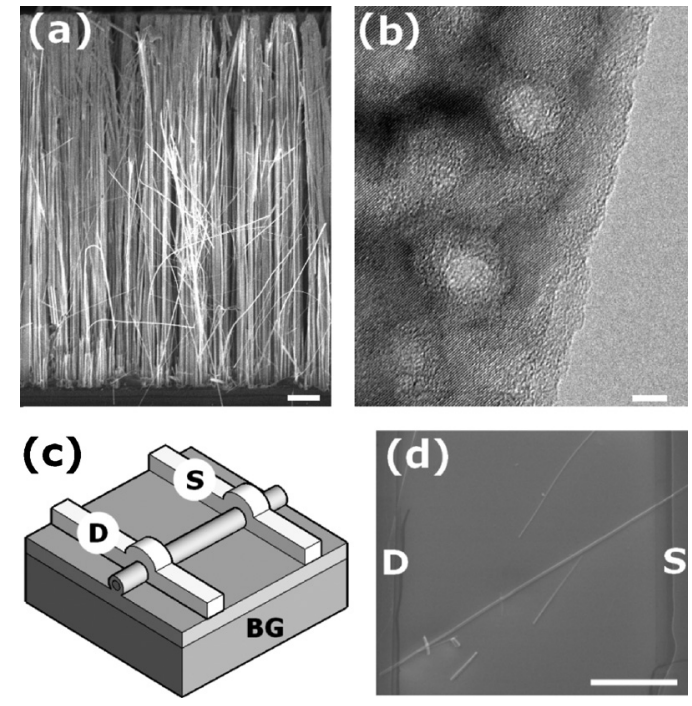

FIG. 1. (a) Scanning electron micrograph of a SiNW array, prepared by MACE. (b) Transmission electron micrograph of a single SiNW. (c) Schematic diagram of a back-gated SiNW FET. Source (S), drain (D), and backgate (BG) contacts are marked. (d) Scanning electron micrograph of a SiNW FET. The scale bars are $10 \mu \mathrm{m}, 5 \mathrm{~nm}$, and $10 \mu \mathrm{m}$, respectively. 

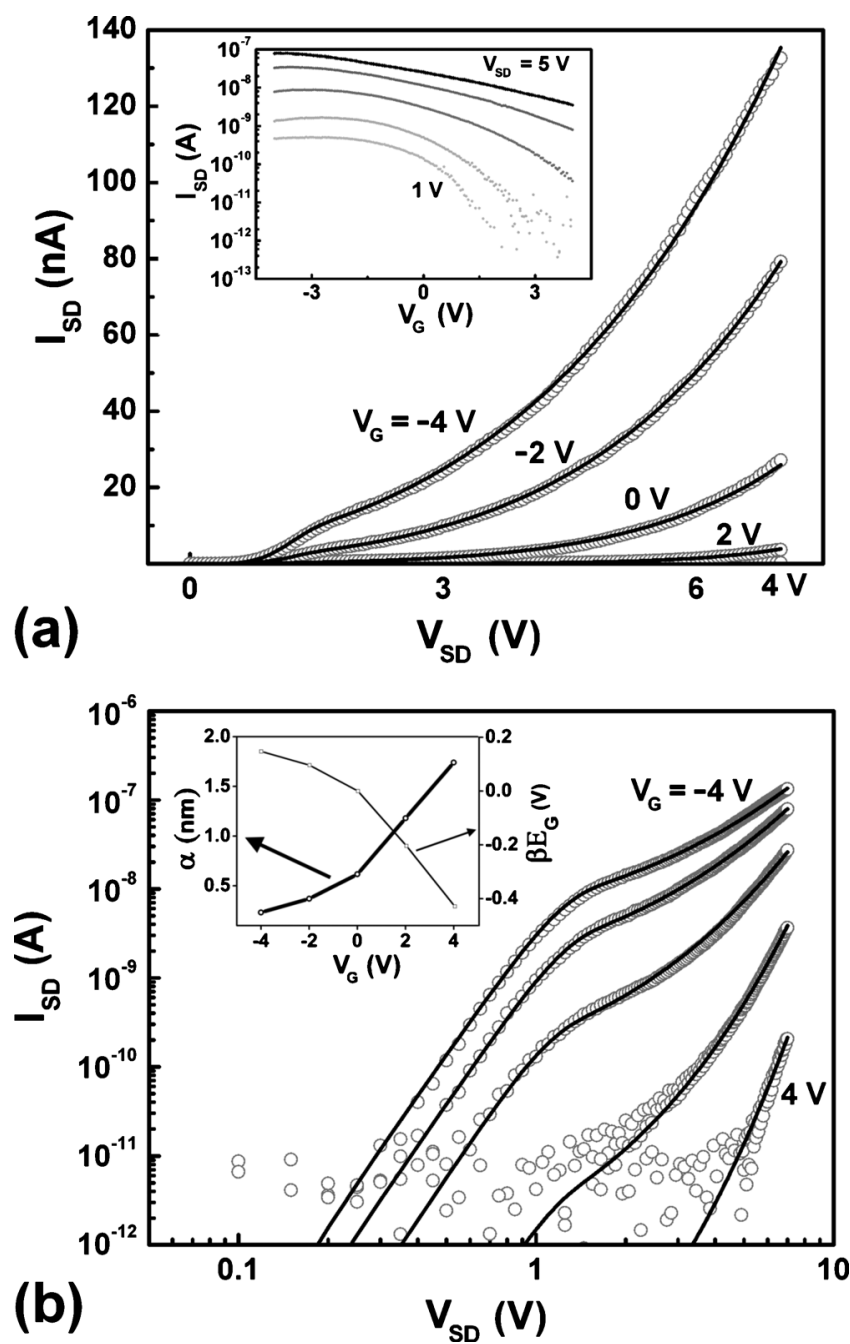

FIG. 2. (a) Experimental (circles) data and theoretical fits (solid lines) for the $I_{S D^{-}} V_{S D}$ output characteristics of a SiNW FET. The inset shows the experimental $I_{S D^{-}} V_{G}$ transfer characteristics, as $V_{S D}$ is varied from 5 to $1 \mathrm{~V}$ in $1 \mathrm{~V}$ steps. (b) Experimental data and theoretical fits on a log-log scale. The inset shows $\alpha$ and $\beta E_{G}$ vs $V_{G}$, extracted from the theoretical fits.

and acts as the back-gate. SiNWs dispersed in IPA were deposited onto this substrate by allowing a drop of solution to evaporate on the surface. The sample was then oxidized at $900{ }^{\circ} \mathrm{C}$ for $10 \mathrm{~min}$, forming a $\sim 10 \mathrm{~nm}$ thick oxide on the NW and passivating the NW surface. An optical lithography process was used to define source/drain contacts on a single, selected NW. After a brief buffered-HF etch of the contact area, $40 \mathrm{~nm}$ of $\mathrm{Ti}$ was evaporated thermally, followed by lift-off of excess Ti. The device was then rapid-thermal annealed at $700{ }^{\circ} \mathrm{C}$ for $30 \mathrm{~s}$ to form a $\mathrm{Ti}_{\mathrm{x}} \mathrm{Si}_{\mathrm{y}}$ silicide contact. A 100-nm-thick Al capping layer was then evaporated thermally over the contact regions to complete the contacts. Figure 1(d) shows a scanning electron micrograph of a device.

Figure 2(a) shows the room temperature $I_{S D}-V_{S D}$ output characteristics (circles) of a "normally-on" FET at various back-gate voltages $V_{G}$. The device was fabricated using a single $p$-type SiNW, $\sim 100 \mathrm{~nm}$ in diameter. The drain-source separation in this device, corresponding to the channel length, was $\sim 20 \mu \mathrm{m}$. $I_{S D}$ increases in magnitude as $V_{G}$ varies from +4 to $-4 \mathrm{~V}$, indicating $p$-type operation. In this device, there is a threshold drain-source voltage for current flow, $V_{S D T} \sim 1 \mathrm{~V}$, suggesting the presence of SB contacts. Above $V_{S D T}, I_{S D}$ increases rapidly and then weakly-saturates

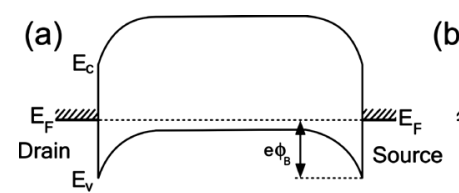

(c)
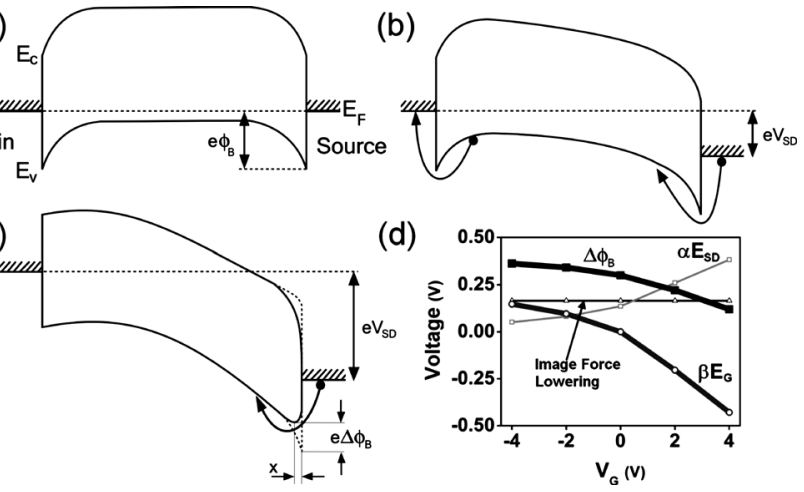

FIG. 3. (a) Energy band diagram of a SB SiNW FET at zero bias. (b) At low bias, thermionic emission of holes occurs across the source and drain SBs. (c) At high bias, SB lowering occurs at the source. Height of $\mathrm{SB} e \phi_{B}$ is lowered by $e \Delta \phi_{B}$ and the valence band edge maximum is shifted a distance $x$ from the contact interface. (d) $\Delta \phi_{B}$, and individual barrier lowering terms from Eq. (2), vs $V_{G}$, at $V_{S D}=4 \mathrm{~V}$.

above $V_{S D} \sim 1.5 \mathrm{~V}$. $I_{S D}$ rises throughout the saturation region, increasing more rapidly at higher $V_{S D}$. As $V_{G}$ varies from +4 to $-4 \mathrm{~V}$, the channel conductance $g_{S D}=\partial I_{S D} / \partial V_{S D}$ increases. The inset to Fig. 2(a) shows the corresponding $I_{S D^{-}} V_{G}$ transfer characteristics. The maximum on-off ratio $I_{o n} / I_{o f f} \sim 1500$, for $V_{S D}=2 \mathrm{~V}$. The peak value of the extrinsic transconductance $g_{m}=\partial I_{S D} / \partial V_{B G}=19 \mathrm{nS}$, at $V_{S D}=5 \mathrm{~V}$ and $V_{B G}=-2 \mathrm{~V}$, and the minimum sub-threshold swing $S$ $=750 \mathrm{mV} /$ decade. Figure 2(b) shows the $I_{S D^{-}} V_{S D}$ characteristics on a log-log plot. The characteristics shift to higher $V_{S D}$ as $V_{G}$ increases from -4 to $+4 \mathrm{~V}$, implying an increase in $V_{S D T}$ with $V_{G}$.

We explain the device characteristics by considering SB lowering at the source contact, as a function of $V_{S D}$ and $V_{G}$. Figure 3 shows schematically the energy band diagram in a $p$-channel SB SiNW FET. At $V_{S D}=V_{G}=0$, SBs lie along the conduction path at both source and drain contacts [Fig. 3(a)]. For $V_{S D}>0 \mathrm{~V}$, the source SB is reverse-biased and the drain $\mathrm{SB}$ is forward-biased. Current flow then consists of holes injected into the NW across the source SB. At small $V_{S D}$, the drain SB is not completely forward-biased and forms a potential barrier along the current path [Fig. 3(b)]. As $V_{S D}$ increases further, the drain SB is fully forward-biased [Fig. 3(c)]. $I_{S D}$ is then controlled solely by the reverse-biased source SB.

We find that the $I_{S D^{-}} V_{S D}$ characteristics in the saturation region (Fig. 2) can be modeled by thermionic emission of holes across the reverse-biased source SB, and lowering of the SB height with $V_{S D}$ and $V_{G}$. We approximate the voltage drop across the SB as a fraction $C$ of the voltage applied across the device. $I_{S D}$ is then given by the equation for thermionic-emission current across a reverse-biased SB: ${ }^{11}$

$$
I_{S D}=S A^{*} T^{2} \exp \left(\frac{-e\left(\phi_{B}-\Delta \phi_{B}\right)}{k T}\right)\left\{\exp \left(\frac{e C V_{S D}}{k T}\right)-1\right\},
$$

where $S$ is the SiNW channel cross-sectional area, $T$ is the absolute temperature, $\phi_{B}$ is the SB height at zero bias [Fig. $3(\mathrm{a})$ ], $e$ is the electronic charge, and $k$ is the Boltzmann constant. $A^{*}$ is the effective Richardson constant. $\Delta \phi_{B}$ represents SB lowering [Fig. 3(c)], and is given by the following expression: 


$$
\Delta \phi_{B}=2\left(\frac{e E_{S D}}{16 \pi \varepsilon_{s}}\right)^{1 / 2}+\alpha E_{S D}+\beta E_{G}
$$

where $\varepsilon_{S}$ is the static semiconductor permittivity, and $E_{S D}$ and $E_{G}$ are the maximum values of the electric field at the source $\mathrm{SB}$, caused by $V_{S D}$ and $V_{G}$, respectively. Here, we have extended the basic model to include $E_{G}$ explicitly. The first term in Eq. (2) gives image force lowering of the SB due to charge near the metal surface. ${ }^{12}$ The second term is a first order approximation of the contribution of $E_{S D}$ to $\mathrm{SB}$ lowering. ${ }^{12,13}$ The final term approximates the direct contribution of $E_{G}$ to SB lowering. The factors $\alpha$ and $\beta$ determine the proportion of SB lowering caused by $E_{S D}$ and $E_{G}$, respectively. Together, $\alpha$ and $\beta$ determine the distance $x$ from the interface to the SB potential maximum [Fig. 3(c)]. Finally, we allow $\alpha$ to vary with $V_{G}$, as the contribution of $V_{S D}$ to SB lowering can vary at different $V_{G}$.

We use Eqs. (1) and (2) to model the experimental $I_{S D^{-}} V_{S D}$ characteristics in the saturation region $\left(V_{S D}\right.$ $>1.5 \mathrm{~V}) . E_{S D}$ is calculated using the depletion approximation, $E_{S D}=\sqrt{\left(2 e N_{a} / \varepsilon_{s}\right)\left(\phi_{B}-C V_{S D}-\xi-k T / e\right)},{ }^{11}$ where $N_{a}$ is the doping density and $\xi$ is the valance band $E_{V}$ to Fermi level $E_{F}$ offset in the SiNW. The theoretical fits to the data (solid lines, Fig. 2) match the data well. The zero-bias SB height, extracted from our fits, is $\phi_{B}=0.4 \mathrm{eV}$. The inset to Fig. 2(b) shows the relationship of $\alpha$ and $\beta E_{G}$ with $V_{G}$. As $V_{G}$ increases from -4 to $+4 \mathrm{~V}, \alpha$ increases from 0.23 to 1.7 $\mathrm{nm}$, and $\beta E_{G}$ reduces from 0.15 to $-0.43 \mathrm{~V}$. The increase in $\alpha$ implies a greater contribution to SB lowering $\Delta \phi_{B}$ by $V_{S D}$ as the FET turns "off" [Eq. (2)]. In contrast, $\beta E_{G}$ reduces as the FET turns off, tending to enhance the SB at positive $V_{G}$. This implies a reduction in $\Delta \phi_{B}$ and an increase in the total SB height $\phi_{B}-\Delta \phi_{B}$. The combined effect of $\alpha$ and $\beta E_{G}$ gives the change in both the magnitude and slope of $I_{S D}$ with $V_{S D}$ and $V_{G}$. Figure 3(d) shows the gate dependence, at constant $V_{S D}=4 \mathrm{~V}$, of the three SB lowering terms constituting Eq. (2), i.e., the image force lowering [first term, Eq. (2)], $\alpha E_{S D}$ and $\beta E_{G}$. In our model, image force lowering does not depend on $E_{G}$ and is constant. The net effect of these terms is a reduction in the magnitude of $\Delta \phi_{B}$ as $V_{G}$ increases, corresponding to reduced SB lowering.
Finally, the low bias region $\left(V_{S D}<1.5 \mathrm{~V}\right)$ may be modeled by assuming that $C$, the proportion of $V_{S D}$ that drops across the $\mathrm{SB}$, increases with $V_{S D}$. This is because at low bias, the drain SB also contributes to the source-drain resistance [Fig. 3(b)]. Furthermore, there may be voltage drops along the SiNW due to potential barriers in the $\mathrm{NW},{ }^{2}$ e.g., if $|\xi|$ is increased locally due to increased surface depletion. As $V_{S D}$ increases, both the drain $\mathrm{SB}$ and any $\mathrm{NW}$ barriers are pulled-up relative to the source Fermi energy and ultimately, the source SB dominates [Fig. 3(c)]. We find that $I_{S D}$ increases very rapidly, with $C=0.044 V_{S D},{ }^{3}$ for $V_{S D}<1.5 \mathrm{~V}$. For $V_{S D}>1.5 \mathrm{~V}$, where the source $\mathrm{SB}$ dominates, $C=0.15$.

In conclusion, we consider the influence of SB lowering in SiNW FETs using NWs prepared by MACE. The reversebiased source SB is lowered by the image-force potential, and by the local electric field caused by both $V_{S D}$ and $V_{G}$. In the saturation region, SB lowering is sufficient to explain both the drain and gate voltage dependence of the device characteristics.

The authors would like to acknowledge valuable discussion with K. Fobelets and M. Green, SiNW preparation by Chuanbo Li, and the support of the E.ON International Research Initiative.

${ }^{1}$ G. Zheng, W. Lu, S. Jin, and C. M. Lieber, Adv. Mater. 16, 1890 (2004). ${ }^{2}$ J. Appenzeller, J. Knoch, M. T. Bjork, H. Riel, H. Schmid, and W. Riess, IEEE Trans. Electron Devices 55, 2827 (2008).

${ }^{3}$ Y. Cui, Q. Wei, H. Park, and C. M. Lieber, Science 293, 1289 (2001).

${ }^{4}$ A. I. Hochbaum, R. Chen, R. D. Delgado, W. Liang, E. C. Garnett, M. Najarian, A. Majumdar, and P. Yang, Nature (London) 451, 163 (2008).

${ }^{5}$ E. Garnett and P. Yang, Nano Lett. 10, 1082 (2010).

${ }^{6}$ Y. Wu, Y. Cui, L. Huynh, C. J. Barrelet, D. C. Bell, and C. M. Lieber, Nano Lett. 4, 433 (2004)

${ }^{7}$ Z. Gao, A. Agarwal, A. D. Trigg, N. Singh, C. Fang, C. H. Tung, Y. Fan, K. D. Buddharaju, and J. Kong, Anal. Chem. 79, 3291 (2007).

${ }^{8}$ K. Q. Peng, Y. J. Yan, S. P. Gao, and J. Zhu, Adv. Mater. 14, 1164 (2002).

${ }^{9}$ M. Zaremba-Tymieniecki, C. Li, K. Fobelets, and Z. A. K. Durrani, IEEE Electron Device Lett. 31, 860 (2010).

${ }^{10}$ K. Peng, X. Wang, and S. T. Lee, Appl. Phys. Lett. 92, 163103 (2008).

${ }^{11}$ E. H. Rhoderick and R. H. Williams, Metal-Semiconductor Contacts, 2nd ed. (Clarendon, Oxford, 1988).

${ }^{12}$ J. M. Andrews and M. P. Lepselter, Solid-State Electron. 13, 1011 (1970).

${ }^{13}$ G. H. Parker, T. C. McGill, C. A. Mead, and D. Hoffman, Solid-State Electron. 11, 201 (1968). 